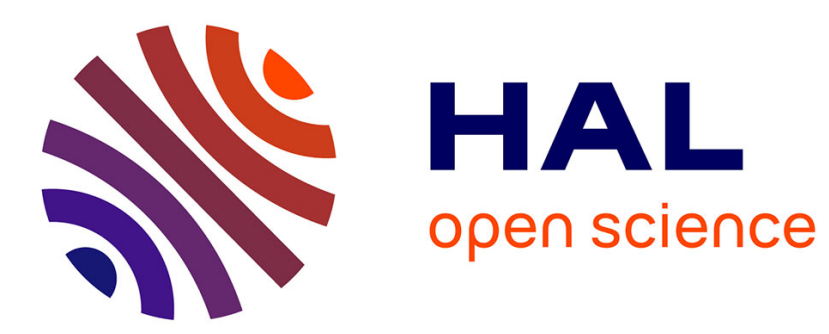

\title{
Dopamine deficiency increases synchronized activity in the rat subthalamic nucleus.
}

\author{
Alessandra Lintas, Isabella G. Silkis, Lavinia Albéri, Alessandro E. P. Villa
}

\section{To cite this version:}

Alessandra Lintas, Isabella G. Silkis, Lavinia Albéri, Alessandro E. P. Villa. Dopamine deficiency increases synchronized activity in the rat subthalamic nucleus.. Brain Research, 2012, 1434, pp.14251. 10.1016/j.brainres.2011.09.005 . inserm-00851266

\section{HAL Id: inserm-00851266 https://www.hal.inserm.fr/inserm-00851266}

Submitted on 13 Aug 2013

HAL is a multi-disciplinary open access archive for the deposit and dissemination of scientific research documents, whether they are published or not. The documents may come from teaching and research institutions in France or abroad, or from public or private research centers.
L'archive ouverte pluridisciplinaire HAL, est destinée au dépôt et à la diffusion de documents scientifiques de niveau recherche, publiés ou non, émanant des établissements d'enseignement et de recherche français ou étrangers, des laboratoires publics ou privés. 


\title{
Dopamine Deficiency Increases Synchronized Activity in the Rat Subthalamic Nucleus
}

\author{
Alessandra Lintas ${ }^{\mathrm{a}, \mathrm{b}, \mathrm{c}}$, Isabella Silkis ${ }^{\mathrm{d}}$, Lavinia Alberi ${ }^{\mathrm{a}}$, Alessandro E.P. Villa ${ }^{\mathrm{e}}$ \\ ${ }^{a}$ Dept. of Medicine/Unit of Anatomy, University of Fribourg, Switzerland \\ ${ }^{b}$ Neuroheuristic Res. Group, Information Science Inst., Univ. of Lausanne, Switzerland \\ ${ }^{c}$ The Schulich School of Medicine, University of Western Ontario, London, Canada \\ ${ }^{d}$ Institute of Higher Nervous Activity and Neurophysiology, Russian Academy of Sciences, Moscow, Russia \\ ${ }^{e}$ INSERM U836; Grenoble Inst. of Neuroscience; Université Joseph Fourier, Eq7 Nanomédecine et Cerveau, Grenoble, France
}

\begin{abstract}
Abnormal neuronal activity in the subthalamic nucleus (STN) plays a crucial role in the pathophysiology of Parkinson's disease (PD). In this study we investigated changes in rat STN neuronal activity after 28 days following the injection of 6-OHDA in the substantia nigra pars compacta (SNc). This drug provoked a lesion of SNc that induced a dopamine (DA) depletion assessed by changes in rotating capacity in response to apomorphine injection and by histological analysis. By means of extracellular recordings and waveshape spike sorting it was possible to analyze simultaneous spike trains and compute the crosscorrelations. Based on the analysis of the autocorrelograms we classified four types of firing patterns: regular (Poissonian-like), oscillatory (in the range 4$12 \mathrm{~Hz}$ ), bursty and cells characterized by a long refractoriness. The distribution of unit types in the control ( $\mathrm{n}=61)$ and lesioned $(n=83)$ groups was similar, as well as the firing rate. In 6-OHDA treated rats we observed a significant increase (from $26 \%$ to $48 \%$ ) in the number of pairs with synchronous firing. These data suggest that the synchronous activity of STN cells, provoked by loss of DA cells in SNc, is likely to be among the most significant dysfunctions in the basal ganglia of Parkinsonian patients. We raise the hypothesis that in normal conditions, DA maintains a balance between funneling information via the hyperdirect corticosubthalamic pathway and parallel processing through the parallel cortico-basal ganglia-subthalamic pathways, both of which are necessary for selected motor behaviors.
\end{abstract}

Keywords: Firing pattern, Basal Ganglia, 6-OHDA-lesioned rats, Parkinson's disease

\section{Introduction}

The subthalamic nucleus (STN) is a part of the cortico-basal ganglia-thalamocortical circuit and abnormal activity of STN plays a crucial role in the pathophysiology of Parkinson's disease (PD) (DeLong and Wichmann, 2007), which is a progressive neurodegenerative disorder manifested by tremor, rigidity, akinesia and bradykinesia. Deep Brain Stimulation (DBS) of STN in Parkinsonian patients, which produces a reversible suppression of its activity, alleviates most of the neurological PD symptoms (Benabid et al., 1994; Limousin et al., 1998). Bilateral STN DBS improved behavioral performance in 6-OHDA lesioned rats (Li et al., 2010).

The STN receives topographically organized inputs from the cerebral cortex, and it provides the major glutamatergic excitation to the output nuclei of the basal ganglia (BG), which are the substantia nigra pars reticulata $(\mathrm{SNr})$ (Villa and Lorenzana, 1997) and internal part of the globus pallidus (GPi) (entopeduncular nucleus in rodents, EPN) (Parent and Hazrati, 1995a,b). Since cortico-basal ganglia circuits are organized in parallel channels, sensory information flow from functionally distinct cortical areas remains segregated within the striatum and through its direct projections to BG output structures (Utter and Basso, 2008). Experimental data indicate that such segregation is only partly maintained in the STN (Kolomiets et al., 2001). However, the STN is in a strategic position to exert a prominent control over the BG-related motor functions since it integrates the somatic motor information from various cortical/subcortical brain areas (including the motor cortex, thalamus and pedunculopontine nucleus) (Takada et al., 1988). Figure 1 illustrates the cortico-BG-thalamocortical circuit influenced by dopamine (DA) neurons of substantia nigra pars compacta $(\mathrm{SNc})$.

Understanding the mechanism of dopamine (DA) influence on the neural circuit involving the BG and STN is important for understanding disturbances in motor behavior and the development of possible therapies for PD. The functional role of DA is complex due to the heterogeneity of neurons within the STN and BG nuclei, presence of dopaminergic D1/D5 or D2/D3 receptors on diverse cell types and their terminals and opposite modulatory effects provoked by DA on different types of pre- and postsynaptic receptors (Sil'kis, 2005). Injection of DA or of D1 receptor agonists into the STN induced an increase in firing rate of the majority of STN neurons in both normal and 6-OHDA rats, but systemic administration of apomorphine (which is a non-selective DA agonist of both D1-like and D2like receptors) provoked a decrease in the firing rate of STN neurons in rats with 6-OHDA lesions (Ni et al., 2001).

Investigation in rat and nonhuman primate models (Bergman et al., 1994; Vila et al., 2000; Tai et al., 2003; Breit et al., 2007) and human PD patients (Pogosyan et al., 2010) showed that the death of DA cells in the SNc may provoke changes of the fir- 


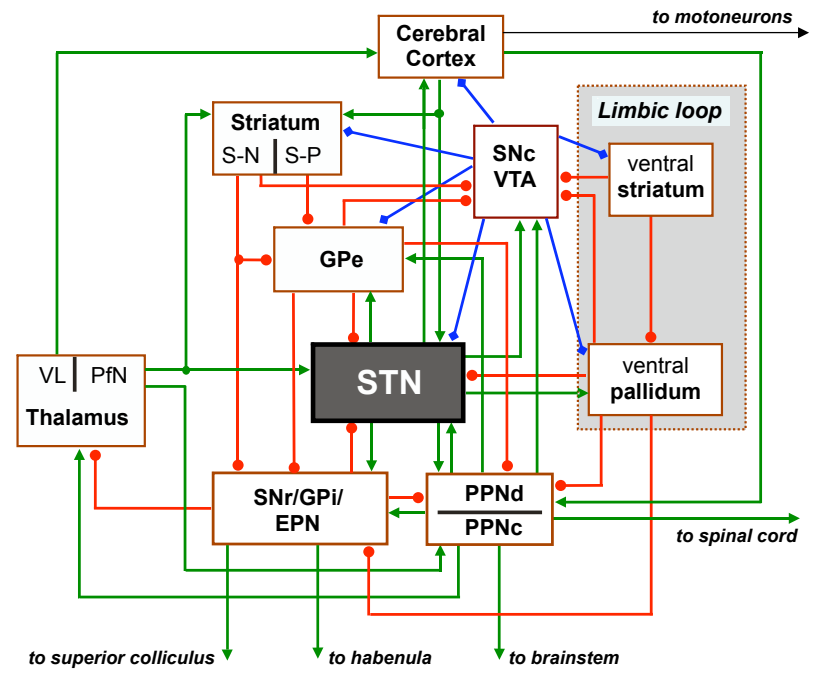

Figure 1: GPe and GPi, external and internal segment of the globus pallidus; D1 and D2, dopaminergic receptors; S-N and S-P, striatonigral and striatopallidal cells; $\mathrm{SNr}$ and SNc, substantia nigra pars reticulata and compacta; STN, subthalamic nucleus; PfN, parafascicular thalamic nucleus, PPNd and PPNc, pars dissipate and compacta of pedunculopontine nucleus; VL, ventral lateral nucleus; VTA, ventral tegmental area. Connections ending with an arrow (green) correspond to excitatory (Glu) projections; ending with a filled circle (red) correspond to inhibitory projections (GABAergic); ending with a filled diamond (blue) indicate the modulatory influence exerted by DA projections of $\mathrm{SNc} / \mathrm{VTA}$.

ing rate and firing pattern of STN neurons and characteristic changes in motor behavior. However, due to the complex organization of BG and the variety of receptors it is difficult to determine the net effects produced by 6-OHDA or MPTP lesion of DA cells in SNc. This is particularly true given the wide range of anesthetics used in acute studies cited in the literature.

In this study, we investigated the changes in rodent STN neuronal activity using multielectrode recordings in the STN performed 28 days after 6-OHDA injection in the SNc. It is important to mention that we performed our recordings under Equithesin anesthesia. The choice of this drug is due to the fact that the other commonly used general anesthetics for rodents (mainly ketamine and urethane) have been reported to exert a strong influence upon the discharge pattern of extracellularly recorded units participating to the cortico-BG-thalamocortical circuit (Villa and Lorenzana, 1997; Ruskin et al., 2003; Hutchison et al., 2004; Chetrit et al., 2009).

We report that in our experimental condition the main effect of 6-OHDA lesion is a significant increase in the synchronous activity of STN cells, which we believe will provoke the main effect exerted by STN in PD models.

\section{Materials and Methods}

\subsection{Subjects and surgical procedure}

Experiments were performed in male Wistar rats weighing $300-350 \mathrm{~g}$. They were kept under controlled conditions of light, temperature and humidity, with food and wa- ter available ad libitum. All animal experiments were carried out in accordance with the UK Animals (Scientific Procedures) Act, 1986 and associated guidelines, the EEC Council Directives (86/609/EEC, OJ L 358, 1, 12 December, 1987) and the Guide for the Care and Use of Experimental Animals (Canadian Council on Animal Care). Rats received atropine sulfate $(0.4 \mathrm{mg} / \mathrm{kg}$, ip $)$ immediately prior to surgery as a prophylactic against respiratory distress. All surgical wounds were infiltrated with Scandicaine 0,5\% (AstraZeneca) for local anesthesia. General anesthesia was induced by Equithesin (3ml/kg,i.p.) (chloral hydrate $4.24 \mathrm{mg}$, sodium pentobarbital $0.97 \mathrm{mg}$, and magnesium sulfate MgSO4 $2.13 \mathrm{mg}$ in $100 \mathrm{ml}$ solution with $11 \%$ ethanol, $42 \%$ propylene glycole $\mathrm{v} / \mathrm{v}$ ) at a dose corresponding to $130 \mathrm{mg} / \mathrm{kg}$ chloral hydrate and $30 \mathrm{mg} / \mathrm{kg}$ pentobarbital (Preda et al., 2008). During the anesthesia the body temperature was monitored and maintained in the range 38$39^{\circ} \mathrm{C}$ by means of a heating pad.

\subsection{6-hydroxydopamine lesion of the $S N c$}

The subjects $(n=8)$ received one injection of $6 \mu l$ of solution in the left substantia nigra pars compacta (SNc) aimed at target stereotaxic coordinates A 3.2; L +2.2; V $7.2 \mathrm{~mm}$ (from lambda and dura) (Paxinos and Watson, 1986). The injected solution contained $12 \mu \mathrm{g}$ 6-OHDA hydrobromide (Sigma, Paris, France) dissolved in $6 \mu \mathrm{l}$ of $0.9 \% \mathrm{NaCl}$ with $0.1 \%$ ascorbic acid (Jouve et al., 2010). It is generally adviced to inject desipramine or pargyline prior to 6-OHDA administration to protect noradrenergic neurons (Breese and Traylor, 1971) but this practice has been questioned (Debeir et al., 2005). There is a nonlinear dose dependent effect of 6-OHDA on noradrenergic neurons, and more recent work has shown that desipramine is not needed at the lower doses of 6-OHDA used in current study (Jouve et al., 2010). Notice that even in case of minor noradrenergic degeneration following 6-OHDA injection, the animal model would be closer to human PD where same degeneration of catcholaminergic cells is likely to be associated to the pathological state (Pillon et al., 1989; Mavridis et al., 1991; Delaville et al., 2011). For control subjects $(n=8)$, an equal volume of vehicle $(6 \mu l$ saline with $0.1 \%$ ascorbic acid) was injected. The 6-OHDA solution were kept on ice $\left(4^{\circ} \mathrm{C}\right)$ and protected from light to minimize oxidation. The injection was made using a stainless steel cannula connected via a polyethylene catheter to a Hamilton microsyringe which was controlled by an infusion pump at a flow rate of $0.1 \mu \mathrm{l} / \mathrm{min}$. At the end of each injection, the syringe was kept in place for additional 5 minutes before being very slowly retracted from the brain, in no less than 5 more minutes.

\subsection{Rotational behavior}

Three weeks after the 6-OHDA-induced lesions were performed, the rats were screened for rotation response to subcutaneous injection of apomorphine $(0.5 \mathrm{mg} / \mathrm{kg}$, s.c., Sigma) (Casas et al., 1999). Five minutes after the apomorphine injection DA-lesioned rats showed more than 20 rotations per 5 min to the controlateral side. One subject did not match this criterion and was discarded from further study. It has previously 
been demonstrated that rats satisfying this criterion are characterized by striatal DA depletion greater than 95\% (Papa et al., 1994).

\subsection{Electrophysiological recordings}

General anesthesia was induced by Equithesin $(3 m l / k g, i . p$.$) .$ The pedal withdrawal reflex was periodically checked and supplemental doses of Equithesin were provided during the whole recording session if necessary. Extracellular single unit recordings in STN (3.6 - $4.2 \mathrm{~mm}$ posterior to bregma, L 2.3-3.7 $\mathrm{mm}$, V 6.5-8.5 $\mathrm{mm}$ from the dura), (Paxinos and Watson, 1986) ipsilateral to the side of injection in SNc (in this study always the left hemisphere), were performed with glass-coated platinumplated tungsten microelectrodes having an impedance in the range $0.5-2 M \Omega$ measured at a frequency of $1 \mathrm{kHz}$ (Frederick Haer \& Co, Bowdoinham, Maine, USA). Signals from the microelectrodes were amplified, filtered $(400 \mathrm{~Hz}-20 \mathrm{kHz})$, viewed on an oscilloscope, and digitally recorded in WAV format (44100 $\mathrm{Hz}$ sampling rate, 16 bit resolution) for computerized offline analysis. Spike sorting of the electrode signal files, based on a template matching algorithm, was able to separate up to three spikes recorded from the same channel (Asai et al., 2005). The time of spike discharges were digitally stored at a time resolution of $1 \mathrm{~ms}$ for later processing.

The first recording session started approximately $90 \mathrm{~min}$ after the end of the surgical preparation. The data were gathered during spontaneous activity, i.e. in the absence of any operatorinduced stimulation, for an interval of 5-10 min. All recordings started at least $15 \mathrm{~min}$ after any supplementary dose of anesthetic and terminated at least 20 min before a new injection, thus assuming the recording conditions corresponded to a steady level of anesthesia.

\subsection{Histological and immunohistological examinations}

Upon completion of the recording session (lasting in total $5-8 h$ ), electrolytic lesions were placed at specific depths of the electrode track using 10 current pulses of $8 \mu \mathrm{A}$ for $7 \mathrm{~s}$ at regular intervals of 10 seconds. Subjects were sacrificed by transcardial perfusion with $100 \mathrm{ml} 0.9 \% \mathrm{NaCl}$ immediately followed by $500 \mathrm{ml}$ of fixative solution (4\% paraformaldehyde in phosphate buffer $0.1 M, \mathrm{pH} 7.3$ ). The brains were removed after perfusion and cut into $40 \mu \mathrm{m}$ sections using a microtome. Sections including SNc were examined for immunohistochemical staining of tyrosine hydroxylase (TH). In sections from 6OHDA treated rats this analysis was aimed at determining the extent of the SNc lesion. Brain slices were immunoreacted with a primary, polyclonal antibody against rat tyrosine hydroxylase (TH, Abcam PLC, Cambridge, UK) and then with a biotinylated goat anti-rabbit IgG (Vector Labs, Burlingame, CA) secondary antibody. The signal was amplified using avidin and biotinylated horseradish peroxidase using an Elite ABC Vectastain Kit (Vector, Burlingame, CA) (Park et al., 2007). Sections including STN and basal ganglia from all subjects were stained with cresyl violet for the reconstruction of the electrode tracks and localization of the electrolytic lesions.

\subsection{Statistical analysis}

Spike trains were analyzed by time series renewal density histograms (Abeles, 1982). Using this technique, all histograms were scaled in rate units (spikes/s) and smoothed after convolution with a moving Gaussian-shaped bin of $5 \mathrm{~ms}$ width. For each histogram, the $99 \%$ confidence limits were calculated, assuming that spike occurrences followed a Poisson distribution. Firing rates of STN neurons of both groups of rats were compared by Mann-Whitney's U-test because of the limited sample sizes we could not test properly the normality of the distributions. A one-tailed Fisher's exact t-test was used to compare the number of each cell type identified and the proportion of significant cross-correlations in both groups of rats.

\section{Results}

\subsection{Effect of 6-OHDA-induced dopamine cell lesion}

Consistent rotational response to the systemic injection of apomorphine $(0.5 \mathrm{mg} / \mathrm{kg})$ was observed in 7 out of 8 treated
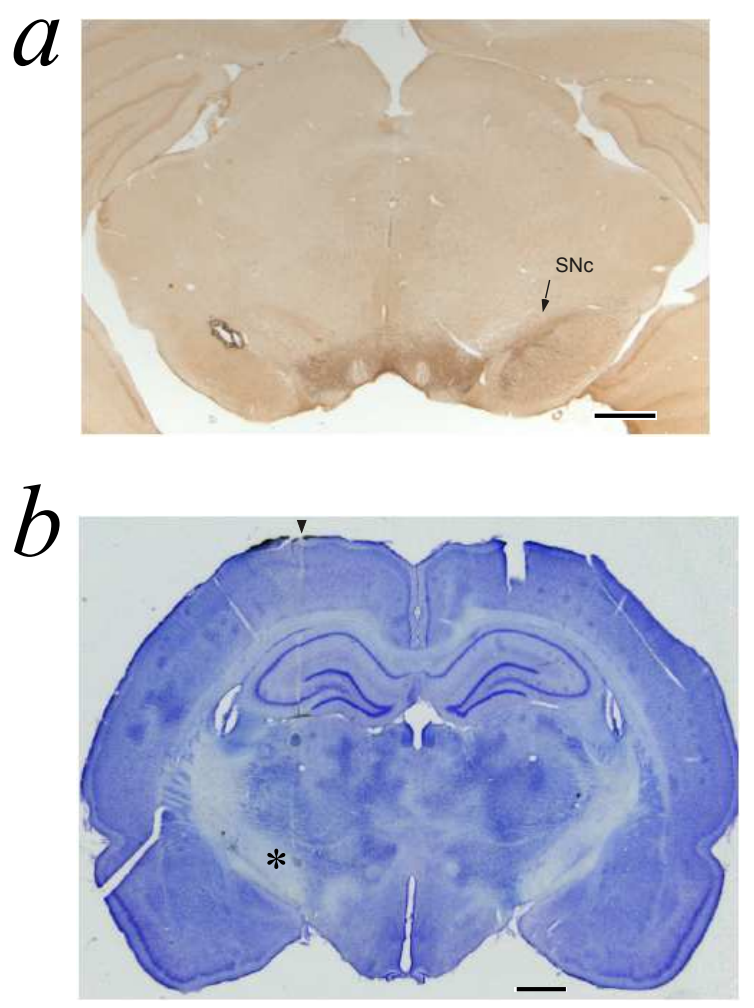

Figure 2: 6-OHDA lesion and reconstruction of the electrode track. Scale bars: $1 \mathrm{~mm}$. (a) Coronal section stained for tyrosine hydroxylase (TH) showing the substantia nigra pars compacta $(\mathrm{SNc})$ at interaural stereotaxic coordinate approximately equal to $\mathrm{Ia}=2.9$ (Paxinos and Watson, 1986). Note the lack of $\mathrm{TH}$ staining in the SNc of the lesioned hemisphere (left) compared to the nonlesioned hemisphere (right). (b) Coronal section, stained with cresyl violet, of the subthalamic nucleus of a control rat in the left hemisphere illustrating the microelectrode penetration at interaural stereotaxic coordinate approximately equal to $\mathrm{Ia}=5.6$. The entrance of the electrode is indicated by arrow and an asterisk is placed to the left of the site of the electrolytic lesion made at the end of the session. A second lesion is visible dorsally in the lateral dorsal thalamic nucleus. 
rats after 21 days from 6-OHDA injection. All rats that were characterized by a positive rotational behavior showed also a complete loss of $\mathrm{TH}$-immunoreactive neurons restricted to the substantia nigra ipsilateral to the injected hemisphere, as exemplified by the microphotographs in Figure $2 \mathrm{a}$.

\subsection{Electrophysiological recordings}

Extracellular single units were recorded in 15 rats anesthetized with Equithesin ( 7 lesioned and 8 control) . We performed a total of 25 electrode tracks (13 tracks in the control group and 12 tracks in the treated group). A total of 144 single units ( $n=61$ in the control group and $n=83$ in the treated group), localized in STN, were characterized by stable firing rate and a stable autocorrelogram, i.e. no significant difference between the begin and the end of the recording session. An additional 94 single units were recorded in the same experiments, but either they did not match the above-mentioned criteria or their location was not in the STN after histological check. Figure 2b illustrates an example of an electrolytic lesion in STN (recording performed in the left hemisphere) and a second lesion performed in the lateral dorsal thalamic nucleus for measurement of tissue retraction necessary for an accurate reconstruction of the recording sites.

\subsubsection{Properties of individual neurons}

The analysis of the autocorrelograms allowed us to define 4 types of firing patterns of the spontaneous activity of STN cells. Units characterized by a significant refractory period were termed "initial prolonged through" (IPT) cells (Fig. 3a). Units that showed a tendency to fire in bursts ("bursting cells", $\mathrm{BC}$ ) were characterized by a hump in the autocorrelogram near time zero (Fig. 3b). Units that presented an autocorrelogram with dampened periodic oscillatory activity were termed "oscillatory cells" (OSC) (Fig. 3c). Units with a flat autocorrelogram formed the "regular" (REG) type class (Fig. 3d).

In the control group type REG units were the most abundant $(40 \%, \mathrm{n}=24)$ followed by the IPT $(23 \%), \mathrm{BC}(21 \%)$ and OSC $(16 \%)$ which were almost equally represented. In 6-OHDA lesioned rats the distribution of the four cell types (Table 1) was not statistically different from the controls (two sided Fischer's exact test, not significant). However, we cannot rule out that

Table 1: Firing rates (median, mean \pm S.E.M.) of the STN units grouped by cell types in control and 6-OHDA treated rats recorded under general anesthesia induced by Equithesin.

\begin{tabular}{cccccc}
\hline Cell Type & & IPT & BC & OSC & REG \\
\hline Control group & & & & & \\
$\mathrm{N}$ & 61 & 14 & 13 & 10 & 24 \\
& $(100 \%)$ & $(23 \%)$ & $(21 \%)$ & $(16 \%)$ & $(40 \%)$ \\
Firing rate & & 6.7 & 1.3 & 5.1 & 1.7 \\
(spikes/s) & & $(6.9 \pm 2.3)$ & $(2.5 \pm 1.5)$ & $(6.1 \pm 2.6)$ & $(2.6 \pm 1.0)$ \\
6-OHDA treated group & & & & & \\
$\mathrm{N}$ & 83 & 26 & 24 & 10 & 23 \\
& $(100 \%)$ & $(31 \%)$ & $(29 \%)$ & $(12 \%)$ & $(28 \%)$ \\
Firing rate & & 6.2 & 2.0 & 4.5 & 2.7 \\
(spikes/s) & & $(6.5 \pm 2.5)$ & $(2.8 \pm 1.5)$ & $(6.7 \pm 4.7)$ & $(2.9 \pm 1.7)$ \\
\hline \hline
\end{tabular}

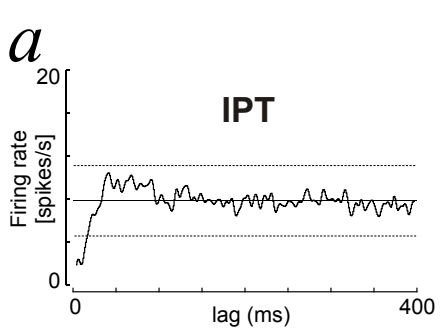

$b_{20}$
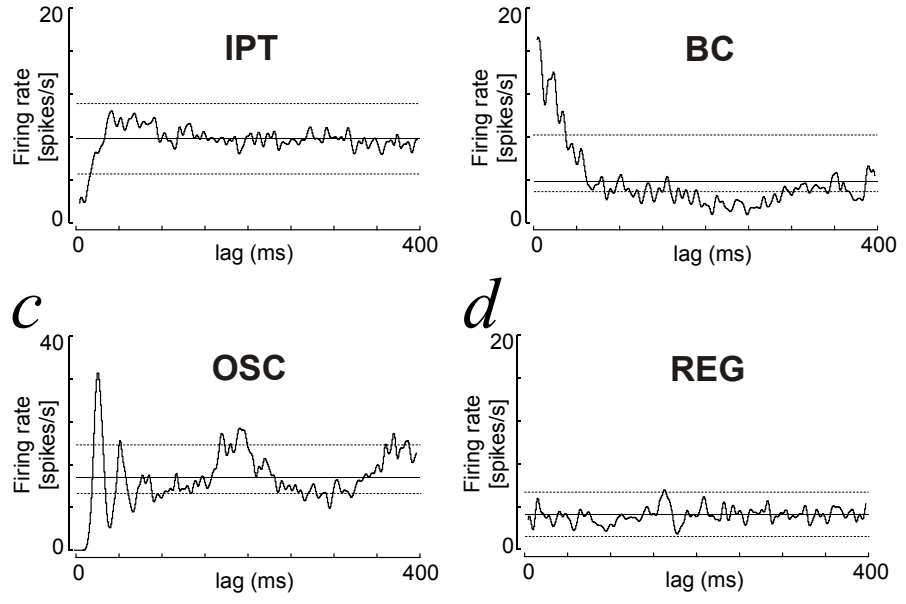

Figure 3: Example of the four specific types of spontaneous firing pattern observed in STN. Autocorrelograms (auto renewal density histograms) with lag ( $m s$ ) on the abscissa and rate (spike/s) on the ordinate. Abscissa is scaled to $400 \mathrm{~ms}$ for all plots. The histograms are smoothed by Gaussian shaped bin of $5 \mathrm{~ms}$ calculated according to Abeles (1982). The dashed lines correspond to $99 \%$ confidence limits. (a) Initial prolonged through cell (IPT), firing at 5.9 spikes/s; (b) Bursting cell (BC), firing at 1.9 spikes/s; (c) Oscillatory cell (OSC) with a period of $62 \mathrm{~ms}$, firing at 7.0 spikes $/ \mathrm{s}$. (d) Regular cell (REG), firing at 1.7 spikes $/$ s;

lack of statistical significance is due to the limited size of our samples; there may have been a trend for a decrease in the relative proportion of REG cells and an increase of IPT and BC cells. The firing rate of IPT and OSC was significantly higher than that of BC and REG types, for both groups of rats (Mann Whitney's U-test; $U=609, z=2.07, p<0.05$ for the controls and $U=992.5, z=3.02, p<0.01$ for the lesioned). However, the DA depletion did not alter the firing rate of either cell type (Mann-Whitney's U-test, $p>0.05$ for either cell type).

\subsubsection{Interaction between pairs of cells}

We could record overall 100 pairs of cells located in the STN, 42 in normal and 58 in 6-OHDA treated rats. All cell pairs were simultaneously recorded from the same electrode tip. The interaction types were classified according to the shape of the crosscorrelogram densities (CRD) calculated according to Abeles (1982). The independence of firing of a pair of units corresponded to a flat cross-correlogram with statistical fluctuations within the limits of significance (e.g., Fig. 4f). Correlograms with a symmetrical hump centered near zero delay, often referred to as "common input" (CI), indicate that the pair of cells tended to discharge in synchrony (e.g., Fig. 4c).

The classes of correlograms showing significant deviations from flatness were more frequently $(p<0.05)$ observed in 6OHDA treated rats $(38 / 58,66 \%)$ than in control subjects $(17 / 42$, $40 \%$ ) (Fig. 5). Most of the significant interactions were of CI type, namely $11 / 42(26 \%)$ in the control group and 28/58 (48\%) in the 6-OHDA treated group. The other curves with significant deviations from flatness were grouped together in the "other" classes of interactions. 

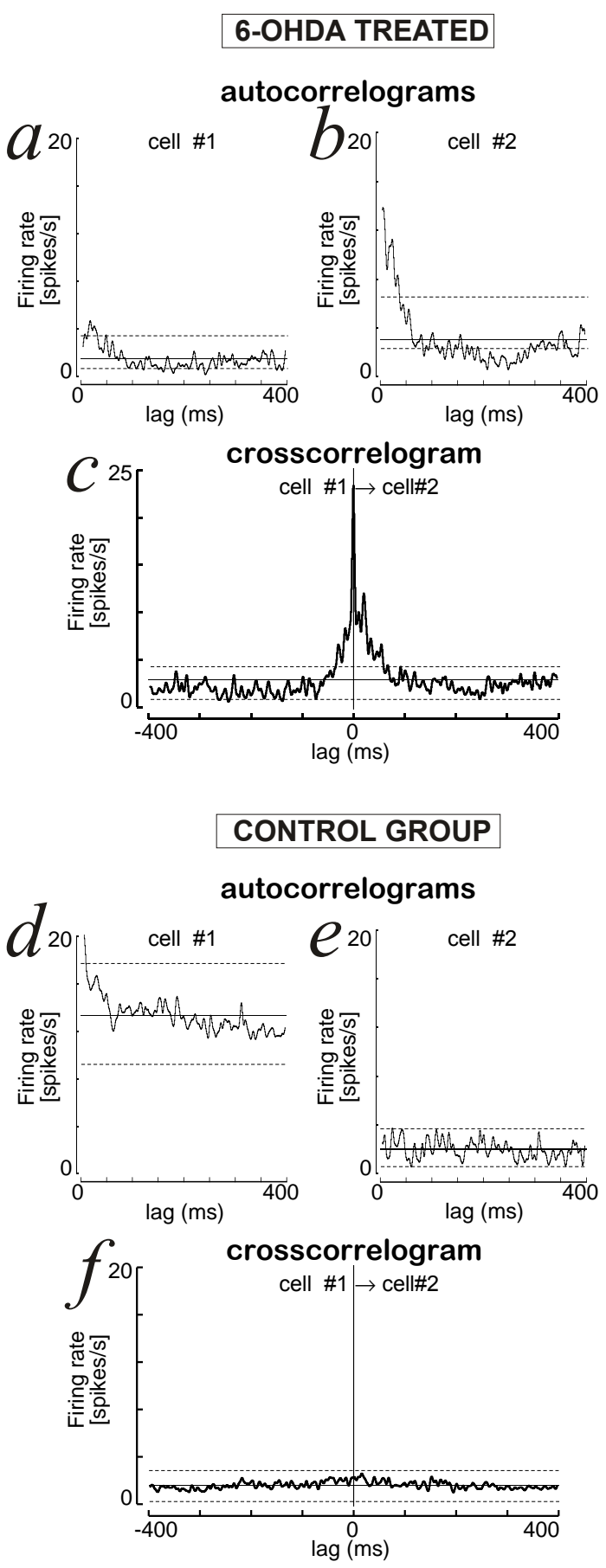

Figure 4: Autocorrelograms and crosscorrelogram of a cell pair recorded in a treated rat (panels a,b,c) and of a cell recorded in a control rat (panels d,e,f). The peak centered near time zero of panel (c) shows synchronous firing of the cells $(a, b)$, referred to as "common input" (CI) type. The flat curve in panel (f) shows no-interaction between cells $(\mathrm{d}, \mathrm{e})$. The curves are smoothed by a Gaussian shaped bin of $5 \mathrm{~ms}$. The firing rates of c\#1 (a) and c\#2 (b) are 1.6 and 1.9 spikes/s, respectively. The firing rates of $\mathrm{c \# 1}$ (d) and c\#2 (e) are 9.2 and 1.1 spikes $/$ s, respectively.

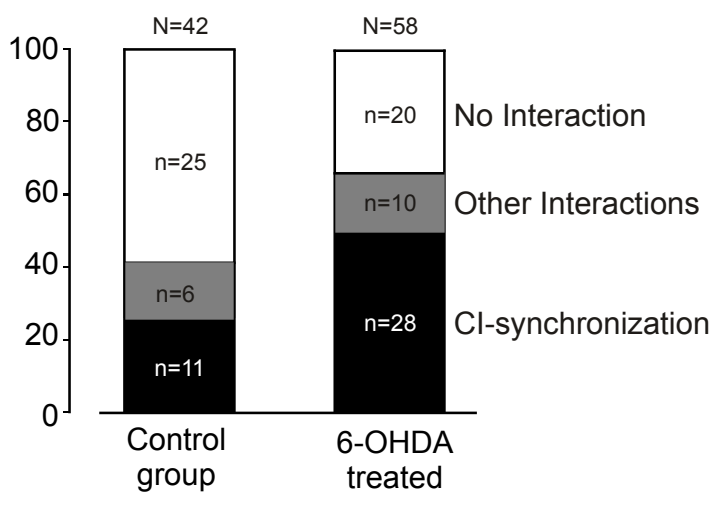

Figure 5: Distribution of crosscorrelogram types ("No Interaction", "Common Input-synchronization", and "Other Interactions" referred to significant interactions other than synchronicity) between pairs of STN units recorded with the same electrode. The ordinate axis is in percentage. The raw numbers of correlograms are indicated by $n$ and $N$.

\section{Discussion}

In the 6-OHDA treated rats of the present study we have demonstrated that the lack of DA does not significantly change the rate and type of firing patterns of STN neurons. We found that cells with initial through in their autocorrelograms (IPT) and cells with oscillatory activity (OSC) were characterized by a similar rate of firing (approximately equal to 6.6 spikes $/ \mathrm{s}$ on average for control and treated rats) which was about twice higher than the rate of the other cell types (approximately equal to 2.7 spikes $/ \mathrm{s}$ on average for control and treated rats). The cell types IPT and OSC represented together 39\% and 43\% of STN units in control and treated rats, respectively. Within this group, the lesion of SNc tended to increase the relative number of IPT firing pattern and to decrease the relative number of OSC. The observation about IPT and OSC might be associated with an increase in recurrent and possibly synchronous feedforward inhibition provided by the loop STN-GPe-STN (Fig. 1) and by the facilitation of the inhibitory interneurons within the STN. The loop STN-GPi-STN can also support recurrent inhibition (Fig. 1).

The stronger STN firing the more effective would be the excitatory effect on the GPe and GPi and subsequent recurrent inhibition of STN neurons as suggested by administration of the D1/D2 agonist apomorphine (Ruskin et al., 2003; Chetrit et al., 2009). In this case the net effect on the firing rate would very small, if any, given an increase in the strength of inhibition of STN neurons following a rise in their activity. In order to understand our results we must also consider the interdependency of the activity of STN and GPe given their strong interconnections. STN and GPe could constitute a kind of central pacemaker modulated by striatal inhibition of GPe neurons responsible for synchronized oscillatory activity in the normal and pathological BG (Plenz and Kital, 1999). DA denervation of the striatum and extrastriatal BG is likely to alter recurrent activity within this circuit thus leading to pathological activity that resonates throughout the BG and motor system (Bevan et al., 2006). The activity of GPe is strongly dependent on fir- 
ing of striatopallidal neurons which mainly express D2 receptors, and give rise to the indirect pathway through the BG. We assume that this pathway is the one that determines mainly the activity of STN neurons. In case of loss of DA in the striatum, the STN could be disinhibited via the indirect pathway due to stronger activity of striatopallidal cells and suppression of firing of the GABAergic GPe cells projecting to the STN (Sil'kis, 2005). This hypothesis is supported by the fact that a high DA concentration is necessary for the activation of $\mathrm{D} 2$ receptors on striatopallidal cells (Williams and Millar, 1990). which in turn would affect STN activity according to the intensity of DA cell lesion.

Our results are partially in disagreement with previous studies that reported an increase in the firing rate of STN neurons with more irregular and bursty-firing patterns of STN neurons as well as significant decrease in relative amount of tonic neurons (Breit et al., 2007). In addition, many existing studies show contrasting tendencies to increasing or to decreasing firing rate in STN after lesion of the SNc (Hollerman and Grace, 1992; Hassani et al., 1996; Vila et al., 2000; Ni et al., 2001; Magill et al., 2001). We observed a decrease in the relative number of OSC cell types, in contrast with an increase in the incidence of slow oscillations $(0.3-2.5 \mathrm{~Hz}$ ) reported by Walters et al. (2007) after dopamine cell lesion in urethane-anesthetized rats. A possible source of discrepancy with previous studies may arise from the use of different anesthesia. We avoided the use of ketamine because we had previously observed that this non-competitive antagonist of NMDA receptors produces a massive change of firing patterns in the $\mathrm{SNr}$ of control animals (Villa and Lorenzana, 1997). We have also avoided the use of urethane because of urethane-induced slow wave cortical activity that alters significantly the analysis of correlations between spike trains (Manns et al., 2000a,b; Jones, 2004; Kasanetz et al., 2008). Our finding that IPT and regular spiking neurons were the commonest types of firing under Equithesin anesthesia appears in agreement with recordings performed in the STN of unanesthetized 6-OHDA treated rats that shown also a decrease in the number of oscillatory units (Kreiss et al., 1997; Allers et al., 2000). This indicates that the active metabolites of Equithesin are unlikely to exert a strong influence upon the discharge patterns of STN units. Accordingly, Equithesin anesthesia could be viewed as a good control condition for studies in anesthetized rats.

The main finding of this study was that in the 6-OHDA treated rats pairs of simultaneously recorded adjacent STN units fired significantly more often in synchrony. A significant increase in cross-correlations between STN neurons has also been observed following a lesion of GPe without 6-OHDA treatment (Ryan et al., 1992). This result is comparable with ours because a lesion of GPe decreases STN inhibition, and DA deficiency leads to STN disinhibition via the indirect pathway through the BG (Fig. 1) . In the rat the neurons of STN are characterized by an important dendritic field around the cell bodies, thus suggesting that excitatory recurrent collaterals could represent the element of circuitry subserving the synchronization of closely spatially related neurons (Hammond et al., 1983). Strong synchronization in STN cell firing could also modify

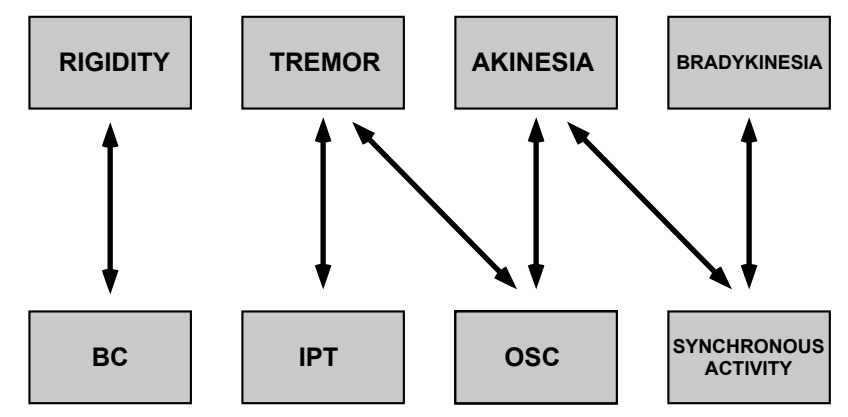

Figure 6: Dependencies between human symptoms and firing pattern parameters. Adapted from Chibirova (2006).

propagation of signals from the neocortex to the output BG nuclei. It is known that hyperdirect cortico-subthalamic pathway may funnel the information whereas the indirect cortico-striatosubthalamic pathway may process the information through parallel loops (Parent et al., 2000). Indeed, excitatory responses to stimulation of diverse cortical areas were found in distinct striatal territories, and none of the cells responded to two cortical stimulation sites (Kolomiets et al., 2001). In contrast, the existence of specific patterns of convergence of information flow from functionally distinct cortical areas in the STN allows interactions between parallel channels (Kolomiets et al., 2003; Bar-Gad et al., 2003).

It is widely believed that Parkinsonian symptoms are most likely caused by abnormal synchronous oscillating neuronal activity within the BG (Bergman and Deuschl, 2002; Avila et al., 2010). Akinesia and hypokinesia could be caused by abnormal hypoactivity of the GPe neurons and subsequent excessive inhibition of BG targets (Wichmann and DeLong, 1996). Recordings in human STN of PD patients during DBS neurosurgery (Chibirova, 2006) showed that the same firing patterns observed in our study (i.e., IPT, BC, OSC and REG represented $33 \%, 19 \%, 15 \%, 33 \%$, respectively) in a proportion similar to the one in 6-OHDA treated rats (Table1). Chibirova (2006) demonstrated that the clinical symptoms were associated to specific features in the firing pattern observed in the STN cells of PD patients (Fig. 6). Motor impairments typically associated to clinical symptoms in human PD patients have been observed in rodents treated with MPTP or 6-OHDA. A complete lesion of SNc by 6-OHDA injection impaired dramatically (by $80 \%$ ) the stepping of the contralateral paw (Barnéoud et al., 2000). Animals with more than $95 \%$ bilateral SNc lesion demonstrated bradykinesia symptoms (Sakai and Gash, 1994) and $\approx 75 \%$ DA cells loss produced forelimb akinesia manifested in stepping deficit (Tseng et al., 2005). It is important to note that akinesia induced by 6-OHDA could be restored to normal locomotion by DBS of the STN, which caused a decrease in firing rate and burst firing in the contralateral side of STN (Shi et al., 2006) and stepping performance in MPTP-treated mice was significantly improved by administration of L-DOPA (Blume et al., 2009). Animal models of PD can never reproduce the entire range of symptoms observed in human patients but they have proven to be valuable for the investigation of the subsequent mechanisms. 
It is interesting to compare our results, which emphasize the increase in STN synchronous activity in 6-OHDA treated rats, with those of Chibirova (2006) who found a significant association between synchronous firing in the STN of PD patients with akinesia and bradykinesia but not with tremor and rigidity (Figure 6). Given the complexity of the physiopathological mechanisms which generate parkinsonian symptomatology the assumption that each class of clinical signs (tremor, akinesia, rigidity, dyskinesia, bradykinesia) is produced by a specific and separate mechanism was questioned (Gross et al., 1999). We support the hypothesis that synchronous activity of STN neurons is correlated with akinesia because it could promote increase in $\mathrm{SNr}$ cell firing due to simultaneous glutamate release from numerous STN terminals in the SNr and its high concentration in synaptic cleft must promote increase in $\mathrm{SNr}$ cell firing. Increased glutamatergic innervation of the $\mathrm{SNr}$ is known to contribute to the motor deficits in PD, whereas suppression of glutamate release by injection of agonists of group III mGlu receptors into the $\mathrm{SNr}$ exerts functional protection against 6-OHDA lesion (Austin et al., 2010). The same procedure reversed reserpine-induced akinesia (Austin et al., 2010) indirectly suggesting the important role of glutamate concentration in the $\mathrm{SNr}$ for akinesia. It has been proposed that the normal dopaminergic system supports segregation of the functional subcircuits of the BG (Bergman et al., 1998) and that the BG-thalamocortical loop dynamically modulates the degree of correlation of neuronal activity in order to select the proper motor behavior (Yasoshima et al., 2005; Gale et al., 2009). We suggest that dopaminergic input is necessary to maintain a balance between funneling and parallel processings, and changes in DA concentration exert modulatory role upon switching between one to the other mode of transmission.

In conclusion, the finding of increased synchronicity between spatially adjacent STN neurons in 6-OHDA treated rats and the careful analysis of the cell types defined by their firing patterns offer a new insight to the mechanisms of motor disturbances in PD.

\section{Acknowledgments}

The Authors wish to thank Marguerite Kaczorowski for her technical assistance throughout the histological procedures and the reviewers whose comments have contributed to improve the clarity of the paper. We thank A.L. Benabid, S. Chabardes, T. Aksenova, O.K. Chibirova and the neurosurgery and neurology teams of the C.H.U. Grenoble for giving access to the results gathered in human PD patients.

\section{References}

Abeles, M., 1982. Quantification, smoothing, and confidence limits for singleunits' histograms. J Neurosci Methods 5, 317-325.

Allers, K.A., Kreiss, D.S., Walters, J.R., 2000. Multisecond oscillations in the subthalamic nucleus: effects of apomorphine and dopamine cell lesion. Synapse 38, 38-50.

Asai, Y., Aksenova, T., Villa, A.E.P., 2005. On-line real-time oriented application for neuronal spike sorting with unsupervised learning. Lecture Notes in Computer Science 3696, 109-114.
Austin, P.J., Betts, M.J., Broadstock, M., O’Neill, M.J., Mitchell, S.N., Duty, S., 2010. Symptomatic and neuroprotective effects following activation of nigral group iii metabotropic glutamate receptors in rodent models of parkinson's disease. Br J Pharmacol 160, 1741-1753.

Avila, I., Parr-Brownlie, L.C., Brazhnik, E., Castañeda, E., Bergstrom, D.A., Walters, J.R., 2010. Beta frequency synchronization in basal ganglia output during rest and walk in a hemiparkinsonian rat. Exp Neurol 221, 307-319.

Bar-Gad, I., Heimer, G., Ritov, Y., Bergman, H., 2003. Functional correlations between neighboring neurons in the primate globus pallidus are weak or nonexistent. J Neurosci 23, 4012-4016.

Barnéoud, P., Descombris, E., Aubin, N., Abrous, D.N., 2000. Evaluation of simple and complex sensorimotor behaviours in rats with a partial lesion of the dopaminergic nigrostriatal system. Eur J Neurosci 12, 322-336.

Benabid, A.L., Pollak, P., Gross, C., Hoffmann, D., Benazzouz, A., Gao, D.M., Laurent, A., Gentil, M., Perret, J., 1994. Acute and long-term effects of subthalamic nucleus stimulation in parkinson's disease. Stereotact Funct Neurosurg 62, 76-84.

Bergman, H., Deuschl, G., 2002. Pathophysiology of parkinson's disease: from clinical neurology to basic neuroscience and back. Mov Disord 17 Suppl 3, 28-40.

Bergman, H., Feingold, A., Nini, A., Raz, A., Slovin, H., Abeles, M., Vaadia, E., 1998. Physiological aspects of information processing in the basal ganglia of normal and parkinsonian primates. Trends Neurosci 21, 32-38.

Bergman, H., Wichmann, T., Karmon, B., DeLong, M.R., 1994. The primate subthalamic nucleus. ii. neuronal activity in the mptp model of parkinsonism. J Neurophysiol 72, 507-520.

Bevan, M.D., Atherton, J.F., Baufreton, J., 2006. Cellular principles underlying normal and pathological activity in the subthalamic nucleus. Curr Opin Neurobiol 16, 621-628.

Blume, S.R., Cass, D.K., Tseng, K.Y., 2009. Stepping test in mice: a reliable approach in determining forelimb akinesia in mptp-induced parkinsonism. Exp Neurol 219, 208-211.

Breese, G.R., Traylor, T.D., 1971. Depletion of brain noradrenaline and dopamine by 6-hydroxydopamine. Br J Pharmacol 42, 88-99.

Breit, S., Bouali-Benazzouz, R., Popa, R.C., Gasser, T., Benabid, A.L., Benazzouz, A., 2007. Effects of 6-hydroxydopamine-induced severe or partial lesion of the nigrostriatal pathway on the neuronal activity of pallidosubthalamic network in the rat. Exp Neurol 205, 36-47.

Casas, M., Prat, G., Robledo, P., Jané, F., 1999. Rotational behavior in dopamine nigrostriatal denervated rats: effects of a wide range of time intervals between apomorphine administrations. Pharmacol Biochem Behav 62, $481-485$.

Chetrit, J., Ballion, B., Laquitaine, S., Belujon, P., Morin, S., Taupignon, A., Bioulac, B., Gross, C.E., Benazzouz, A., 2009. Involvement of basal ganglia network in motor disabilities induced by typical antipsychotics. PLoS One 4, 1-14.

Chibirova, O., 2006. Interactions fonctionnelles dans les ganglions de la base étudiées par l'enregistrement simultané des activités unitaires discriminées par un algorithme non supervisé de tri de potentiels d'action. Ph.D. thesis. Université Joseph-Fourier - Grenoble I. http://tel.archives-ouvertes.fr/tel00115720/en/.

Debeir, T., Ginestet, L., François, C., Laurens, S., Martel, J.C., Chopin, P., Marien, M., Colpaert, F., Raisman-Vozari, R., 2005. Effect of intrastriatal 6-OHDA lesion on dopaminergic innervation of the rat cortex and globus pallidus. Exp Neurol 193, 444-454.

Delaville, C., Deurwaerdère, P.D., Benazzouz, A., 2011. Noradrenaline and parkinson's disease. Front Syst Neurosci 5, 31-31.

DeLong, M.R., Wichmann, T., 2007. Circuits and circuit disorders of the basal ganglia. Arch Neurol 64, 20-24.

Gale, J.T., Shields, D.C., Jain, F.A., Amirnovin, R., Eskandar, E.N., 2009. Subthalamic nucleus discharge patterns during movement in the normal monkey and parkinsonian patient. Brain Res 1260, 15-23.

Gross, C.E., Boraud, T., Guehl, D., Bioulac, B., Bezard, E., 1999. From experimentation to the surgical treatment of parkinson's disease: prelude or suite in basal ganglia research? Prog Neurobiol 59, 509-532.

Hammond, C., Rouzaire-Dubois, B., Féger, J., Jackson, A., Crossman, A.R., 1983. Anatomical and electrophysiological studies on the reciprocal projections between the subthalamic nucleus and nucleus tegmenti pedunculopontinus in the rat. Neuroscience $9,41-52$.

Hassani, O.K., Mouroux, M., Féger, J., 1996. Increased subthalamic neuronal activity after nigral dopaminergic lesion independent of disinhibition via the 
globus pallidus. Neuroscience 72, 105-115.

Hollerman, J.R., Grace, A.A., 1992. Subthalamic nucleus cell firing in the 6ohda-treated rat: basal activity and response to haloperidol. Brain Res 590, 291-299.

Hutchison, W.D., Dostrovsky, J.O., Walters, J.R., Courtemanche, R., Boraud, T., Goldberg, J., Brown, P., 2004. Neuronal oscillations in the basal ganglia and movement disorders: evidence from whole animal and human recordings. J Neurosci 24, 9240-9243.

Jones, B.E., 2004. Activity, modulation and role of basal forebrain cholinergic neurons innervating the cerebral cortex. Prog Brain Res 145, 157-169.

Jouve, L., Salin, P., Melon, C., Kerkerian-Le Goff, L., 2010. Deep brain stimulation of the center median-parafascicular complex of the thalamus has efficient anti-parkinsonian action associated with widespread cellular responses in the basal ganglia network in a rat model of parkinson's disease. J Neurosci 30, 9919-9928.

Kasanetz, F., Riquelme, L.A., Della-Maggiore, V., O’Donnell, P., Murer, M.G., 2008. Functional integration across a gradient of corticostriatal channels controls up state transitions in the dorsal striatum. Proc Natl Acad Sci U S A 105, 8124-8129.

Kolomiets, B.P., Deniau, J.M., Glowinski, J., Thierry, A.M., 2003. Basal ganglia and processing of cortical information: functional interactions between trans-striatal and trans-subthalamic circuits in the substantia nigra pars reticulata. Neuroscience 117, 931-938.

Kolomiets, B.P., Deniau, J.M., Mailly, P., Ménétrey, A., Glowinski, J., Thierry, A.M., 2001. Segregation and convergence of information flow through the cortico-subthalamic pathways. J Neurosci 21, 5764-5772.

Kreiss, D.S., Mastropietro, C.W., Rawji, S.S., Walters, J.R., 1997. The response of subthalamic nucleus neurons to dopamine receptor stimulation in a rodent model of parkinson's disease. J Neurosci 17, 6807-6819.

Li, X.H., Wang, J.Y., Gao, G., Chang, J.Y., Woodward, D.J., Luo, F., 2010 High-frequency stimulation of the subthalamic nucleus restores neural and behavioral functions during reaction time task in a rat model of parkinson's disease. J Neurosci Res 88, 1510-1521.

Limousin, P., Krack, P., Pollak, P., Benazzouz, A., Ardouin, C., Hoffmann, D., Benabid, A.L., 1998. Electrical stimulation of the subthalamic nucleus in advanced parkinson's disease. N Engl J Med 339, 1105-1111.

Magill, P.J., Bolam, J.P., Bevan, M.D., 2001. Dopamine regulates the impact of the cerebral cortex on the subthalamic nucleus-globus pallidus network. Neuroscience 106, 313-330.

Manns, I.D., Alonso, A., Jones, B.E., 2000a. Discharge profiles of juxtacellularly labeled and immunohistochemically identified gabaergic basal forebrain neurons recorded in association with the electroencephalogram in anesthetized rats. J Neurosci 20, 9252-9263.

Manns, I.D., Alonso, A., Jones, B.E., 2000b. Discharge properties of juxtacellularly labeled and immunohistochemically identified cholinergic basal forebrain neurons recorded in association with the electroencephalogram in anesthetized rats. J Neurosci 20, 1505-1518.

Mavridis, M., Colpaert, F.C., Millan, M.J., 1991. Differential modulation of (+)-amphetamine-induced rotation in unilateral substantia nigra-lesioned rats by alpha 1 as compared to alpha 2 agonists and antagonists. Brain Res $562,216-224$

Ni, Z.G., Bouali-Benazzouz, R., Gao, D.M., Benabid, A.L., Benazzouz, A., 2001. Time-course of changes in firing rates and firing patterns of subthalamic nucleus neuronal activity after 6-ohda-induced dopamine depletion in rats. Brain Res 899, 142-147.

Papa, S.M., Engber, T.M., Kask, A.M., Chase, T.N., 1994. Motor fluctuations in levodopa treated parkinsonian rats: relation to lesion extent and treatment duration. Brain Res 662, 69-74.

Parent, A., Hazrati, L.N., 1995a. Functional anatomy of the basal ganglia. i. the cortico-basal ganglia-thalamo-cortical loop. Brain Res Brain Res Rev 20, 91-127.

Parent, A., Hazrati, L.N., 1995b. Functional anatomy of the basal ganglia ii. the place of subthalamic nucleus and external pallidum in basal ganglia circuitry. Brain Res Brain Res Rev 20, 128-154.

Parent, A., Sato, F., Wu, Y., Gauthier, J., Lévesque, M., Parent, M., 2000. Organization of the basal ganglia: the importance of axonal collateralization. Trends Neurosci 23, 20-27.

Park, Y.S., Jeon, M.F., Lee, B.H., Chang, J.W., 2007. Lesion of subthalamic nucleus in parkinsonian rats : effects of dopamine $\mathrm{d}(1)$ and $\mathrm{d}(2)$ receptor agonists on the neuronal activities of the substantia nigra pars reticulata. J Korean Neurosurg Soc 42, 455-461.
Paxinos, G., Watson, C., 1986. The Rat Brain in Stereotaxic Coordinates. Academic Press, Sydney and Orlando. 2nd edition.

Pillon, B., Dubois, B., Bonnet, A.M., Esteguy, M., Guimaraes, J., Vigouret, J.M., Lhermitte, F., Agid, Y., 1989. Cognitive slowing in parkinson's disease fails to respond to levodopa treatment: the 15-objects test. Neurology 39, $762-768$.

Plenz, D., Kital, S.T., 1999. A basal ganglia pacemaker formed by the subthalamic nucleus and external globus pallidus. Nature 400, 677-682.

Pogosyan, A., Yoshida, F., Chen, C.C., Martinez-Torres, I., Foltynie, T., Limousin, P., Zrinzo, L., Hariz, M.I., Brown, P., 2010. Parkinsonian impairment correlates with spatially extensive subthalamic oscillatory synchronization. Neuroscience 171, 245-257.

Preda, S., Govoni, S., Lanni, C., Racchi, M., Mura, E., Grilli, M., Marchi, M., 2008. Acute beta-amyloid administration disrupts the cholinergic control of dopamine release in the nucleus accumbens. Neuropsychopharmacology 33, 1062-1070.

Ruskin, D.N., Bergstrom, D.A., Tierney, P.L., Walters, J.R., 2003. Correlated multisecond oscillations in firing rate in the basal ganglia: modulation by dopamine and the subthalamic nucleus. Neuroscience 117, 427-438.

Ryan, L.J., Sanders, D.J., Clark, K.B., 1992. Auto- and cross-correlation analysis of subthalamic nucleus neuronal activity in neostriatal- and globus pallidal-lesioned rats. Brain Res 583, 253-261.

Sakai, K., Gash, D.M., 1994. Effect of bilateral 6-ohda lesions of the substantia nigra on locomotor activity in the rat. Brain Res 633, 144-150.

Shi, L.H., Luo, F., Woodward, D.J., Chang, J.Y., 2006. Basal ganglia neural responses during behaviorally effective deep brain stimulation of the subthalamic nucleus in rats performing a treadmill locomotion test. Synapse $59,445-457$.

Sil'kis, I.G., 2005. Possible mechanisms of involvement of the subthalamic nucleus and structures interconnected with this nucleus in movement disorders evoked by dopamine depletion. Usp Fiziol Nauk 36, 66-83.

Tai, C.H., Boraud, T., Bezard, E., Bioulac, B., Gross, C., Benazzouz, A., 2003. Electrophysiological and metabolic evidence that high-frequency stimulation of the subthalamic nucleus bridles neuronal activity in the subthalamic nucleus and the substantia nigra reticulata. FASEB J 17, 1820-1830.

Takada, M., Nishihama, M.S., Nishihama, C.C., Hattori, T., 1988. Two separate neuronal populations of the rat subthalamic nucleus project to the basal ganglia and pedunculopontine tegmental region. Brain Res 442, 72-80.

Tseng, K.Y., Kargieman, L., Gacio, S., Riquelme, L.A., Murer, M.G., 2005. Consequences of partial and severe dopaminergic lesion on basal ganglia oscillatory activity and akinesia. Eur J Neurosci 22, 2579-2586.

Utter, A.A., Basso, M.A., 2008. The basal ganglia: an overview of circuits and function. Neurosci Biobehav Rev 32, 333-342.

Vila, M., Périer, C., Féger, J., Yelnik, J., Faucheux, B., Ruberg, M., RaismanVozari, R., Agid, Y., Hirsch, E.C., 2000. Evolution of changes in neuronal activity in the subthalamic nucleus of rats with unilateral lesion of the substantia nigra assessed by metabolic and electrophysiological measurements. Eur J Neurosci 12, 337-344.

Villa, A.E.P., Lorenzana, V.M.B., 1997. Ketamine modulation of the temporal pattern of discharges and spike train interactions in the rat substantia nigra pars reticulata. Brain Research Bulletin 43, 525 - 535.

Walters, J.R., Hu, D., Itoga, C.A., Parr-Brownlie, L.C., Bergstrom, D.A., 2007. Phase relationships support a role for coordinated activity in the indirect pathway in organizing slow oscillations in basal ganglia output after loss of dopamine. Neuroscience 144, 762-776.

Wichmann, T., DeLong, M.R., 1996. Functional and pathophysiological models of the basal ganglia. Curr Opin Neurobiol 6, 751-758.

Williams, G.V., Millar, J., 1990. Concentration-dependent actions of stimulated dopamine release on neuronal activity in rat striatum. Neuroscience $39,1-$ 16.

Yasoshima, Y., Kai, N., Yoshida, S., Shiosaka, S., Koyama, Y., Kayama, Y., Kobayashi, K., 2005. Subthalamic neurons coordinate basal ganglia function through differential neural pathways. J Neurosci 25, 7743-7753. 